\title{
Annular Lichen Planus On Penis Treated With Topical Pimecrolimus $1 \%$
}

\author{
G Pehlivanov ${ }^{1}$, N Tsekova-Traykovich ${ }^{1}$, I Bakardzhiev ${ }^{2 *}$, A Argirov ${ }^{1}$, G Manolova ${ }^{1}$, I Krasnaliev ${ }^{3}$ \\ ${ }^{1}$ Department of Dermatology and Venereology, Medical University -Sofia, Bulgaria \\ ${ }^{2}$ Medical College, Medical University- Varna, Bulgaria \\ ${ }^{3}$ Department of Pathology, Medical University- Varna, Bulgaria
}

Received: June 15, 2016; Accepted: June 18, 2016; Published: June 22, 2016

*Corresponding author: Assoc. Prof. Ilko Bakardzhiev, Medical College ,Medical University of Varna, Tsar Osvo boditel 84, Bulgaria, Tel: +359 888768

413; E-mail: varna2008@gmail.com

\begin{abstract}
Lichen planus (LP) is an idiopathic inflammatory disease of the skin and mucous membranes. Classical LP is characterized by pruritic, violaceous papules that favor the extremities. Annular lichen planus (ALP) is a long-recognized clinical variant of lichen planus, but is often considered uncommon in occurrence. ALP commonly involves the male genitalia but also has a predilection for intertriginous areas such as the axilla and groin folds. Distal aspects of the extremities, and less commonly the trunk, may also be involved. We report the case of 38 year-old uncircumcised male patient who addressed our clinic for multiple asymptomatic annular lesions on the glans penis and corpus penis and whitish linear bilateral and symmetric lines on buccal mucosa with 2 years duration. During this period the patient was treated several times with antifungal and corticosteroid drugs without improvement. Diagnosis was based on clinical features and histological examination. The patient was treated with topical pimecrolimus $1 \%$ with significant improvement after 3 weeks.
\end{abstract} Penis

Keywords: Lichen Planus (LP); Annular Lichen Planus (ALP);

\section{Introduction}

Lichen planus is a cell-mediated immune response of unknown origin, affecting skin, mucous membranes, scalp and nails. It may be found with other diseases of altered immunity, such as ulcerative colitis, alopecia areata, vitiligo, dermatomyositis, morphea, lichen sclerosis, and myasthenia gravis. The term lichen planus was initially introduced by Erasmus Wilson in 1869 to describe the condition that had been previously named leichen ruber by Hebra. [1] Classical LP is characterized by pruritic, violaceous papules that favor the extremities. [1] It has different variants based on the morphology of the lesions and the site of involvement.

\section{Case Report}

38-year-old man, uncircumcised, heavy smoker was admitted in our clinic. He denied drug abuse and bisexuality. Clinically multiple asymptomatic annular lesions different in size, with slightly raised edge and typically purple to white in color and central portion with skin-color were presented on glans penis and corpus penis (Figure 1). There was no lesion anywhere else on the skin. Whitish linear bilateral and symmetric lines on buccal mucosa were observed. The first lesion was appeared 2 years before as reddish-purple papul which spread peripherally and the central area was resolved. After a period of 6 months the patients noticed the changes on oral mucosa like mild discomfort and altered sensitivity. He was treated several times for Candida balanitis with Fluconazol topical and systemic without clinical improvement. He was also treated with topical corticosteroids for a long time without clinical improvement.

Microscopy and culture spices were negative for Candida spp. and other microorganisms. Serologies for hepatitis B and C, syphilis, and HIV were negative. Histological examination of a biopsy specimen was consistent for Lichen planus. Eipdermal acanthosis with both hyper- orthokeratosis and hypergranulosis were observed. There was also a band-like lymphocytic infiltrate at dermal- epidermal junction with hydropic degeneration of the basal layer with which apoptotic bodies were seen (Figures $2,3,4]$. Direct immune fluorescence was negative. Topical pimecrolimus in combination with immune stimulant drug ( lactofer and colastrum) were prescribed, which led to significant clinical improvement after 3 weeks of treatment (Figures 5, 6).

\section{Discussion}

Annular lichen planus (ALP) is a long-recognized clinical variant of lichen planus, but is often considered uncommon in occurrence. ALP was first reported in literature by Dr. Galloway in 1899 as distinct from lichen planus of annular type. [2] The mechanism of genesis of annular lesion is still unknown, probably expressions of ICAM- 1 and TNF-alpha in the peripheral keratinocytes and dermal infiltrated cells play important role. [3] There is not data for race and gender predisposition in literature. The eruption initially occurred as lichen-papules, then enlarged, and finally developed annular manifestations with raised edges and central cleraing. ALP commonly involves the male genitalia butalso has a predilection for intertriginous areas such as the axilla and groin folds. [4, 5] Distal aspects of the extremities, and less commonly the trunk, may also be involved. Typical lichen papuls 


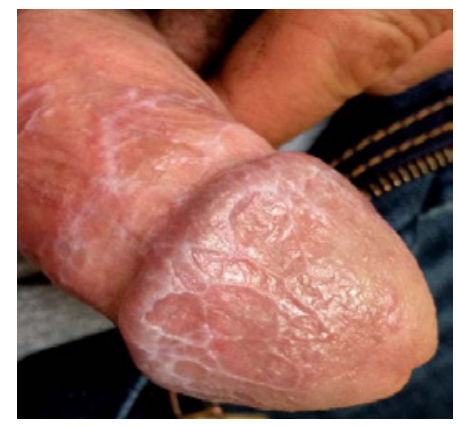

Figure 1: Multiple annular lesions different in size, with slightly raised edge, typically purple to white in color and central portion with skincolor.

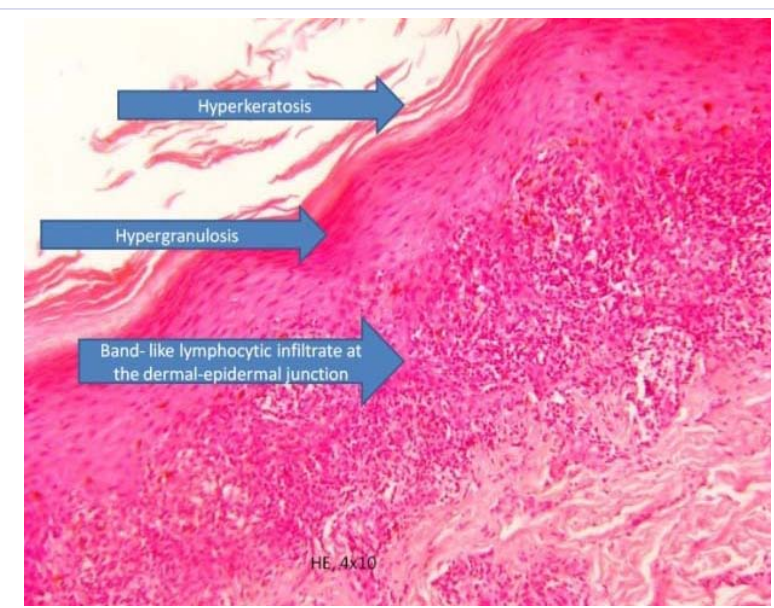

Figure 2: Hyperkeratosis, hypergranulosis, band-like lymphocytic infiltrate at the dermal-epidermal junction, HE $4 \times 10$.

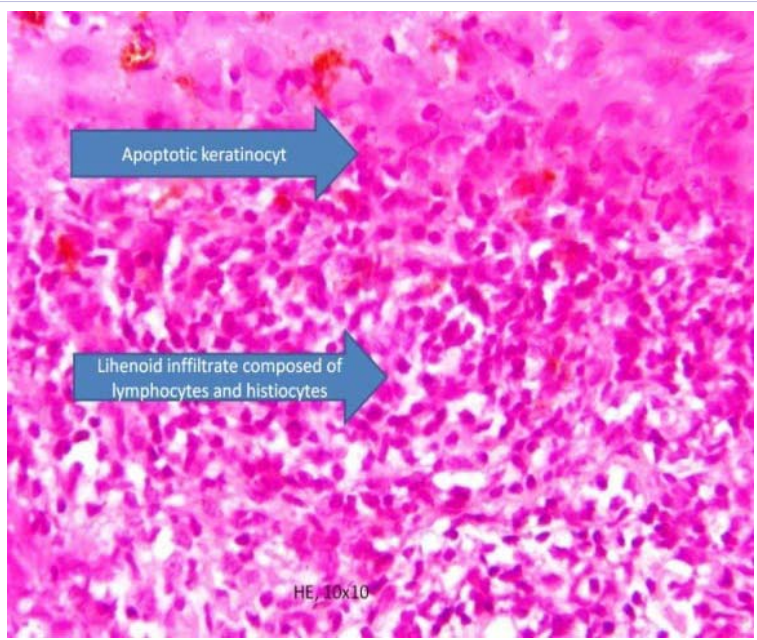

Figure 3: Lichenoid infflitrate composed of lymphocytes and histiocytes, HE $10 \times 10$.

may be observed on the other skin surface. Mucous membranes can also be affected. Although classic LP is pruriginous, ALP proceed without subjective complaints. The main problem is psicho-sexual disorders like in our patients. Diagnosis is based on clinical presentation and histological examination- eipdermal acanthosis with both hyper- orthokeratosis and hypergranulosis, band-like lymphocytic infiltrate at dermal- epidermal junction with hydropic degeneration of the basal layer with apoptotic bodies. There is a wide range of differential diagnoses, particularly Candida balanitis, circinate balanitis in patients with SARA, Reiter Syndrome, balanits with other genesis, granuloma annulare, psoriasis on penis, Lichen sclerosus, syphilis etc. [6, 7] Mid- to high potency topical corticosteroids are the first line

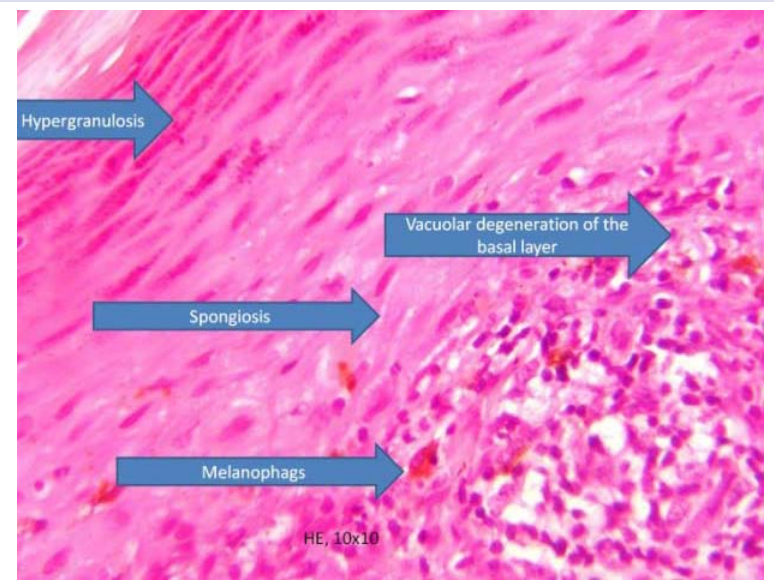

Figure 4: Hypergranulosis, spongiosis, vacuolar degeneration of the basal layer, melanophages, HE 10x10.

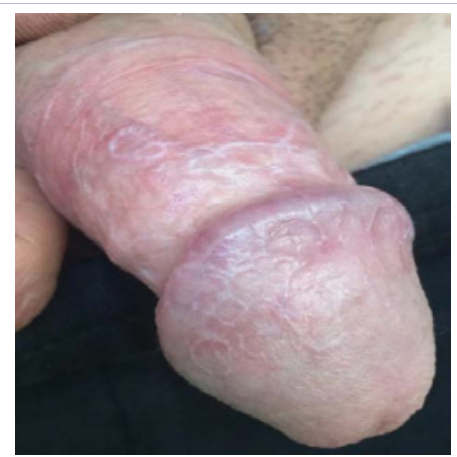

Figure 5: Annular lesions - 3 weeks after treatment with Pimecrolimus $1 \%$.

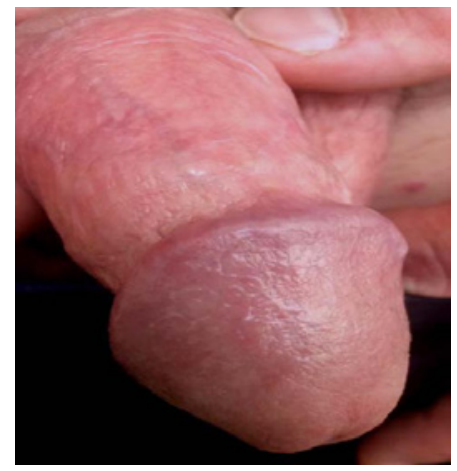

Figure 6: 6 weeks after treatment with Pimecrolimus 1\%. 
treatment in patients with ALP, but when they are use in genital area they hide a risk for atrophy and hemorrhages so maybe use of topical pimecrolimus can be effective. $[8,9,10]$

\section{Conclusion}

This description highlights the importance of patients presenting annular lesion on penis be routinely required to undergo further medical examination for Canddida spec., Sexually transmitted infections and if is necessary to perform the biopsy because the exact diagnosis is basis for proper treatment. To our knowledge this is the first case of ALP reported in literature treated with topical pimecrolimus with significant clinical improvement.

\section{References}

1. Boyd AS, Neldner KH. Lichen planus. J Am Acad Dermatol 1991;25(4):593-619.

2. JMH MacLeod. Annular Lichen planus affecting Penis and Forearm. Proc R Soc Med. 1908;1(Dermatol Sect): 34-35.

3. Ohta Y, Yonemoto K, Asai T, Yaguchi A. Lichen planus annularis: an immunohistochemical study. J Dermtol 1992;19(7):414-419.
4. Reich HL, Nguyen JT, James WD. Annular lichen planus: a case series of 20 patients. J Am Acad Dermatol. 2004;50(4):595-599.

5. Barnette DJ Jr, Curtin TJ, Yeager JK, Corbett DW. Asymptomatic penile lesions. Cutis. 1993;51(2):116-118

6. Teichman JM, Sea J, Thompson IM, Elston DM. Noninfectious penile lesions. Am Fam Physician. 2010;81(2):167-174.

7. Pietrzak A, Chodorowska G, Klijer R, Ciechan J, Bar K, Urban J, et al. A case of lichen planus of the penis mimicking leukoplakia lesions: a review of differential diagnosis. Ann Univ Mariae Curie Sklodowska Med. 2003;58(2):314-319.

8. Fazel N. Cutaneous lichen planus: A systematic review of treatments. J Dermatolog Treat. 2015;26(3):280-283. doi: 10.3109/09546634.2014.933167

9. Atzmony L, Reiter O, Hodak E, Gdalevich M, Mimouni D. Treatments for Cutaneous Lichen Planus: A Systematic Review and Meta-Analysis. Am J Clin Dermatol. 2016;17(1):11-22. doi: 10.1007/s40257-0150160-6.

10. Badri T, Kenani N, Benmously R, Debbiche A, Mokhtar I, Fenniche $\mathrm{S}$. Isolated genital annular lichen planus. Acta Dermatovenerol Alp Pannonica Adriat. 2011;20(1):31-33. 